\title{
On the solvability of discrete nonlinear Neumann problems involving the $p(x)$-Laplacian
}

Aboudramane Guiro ${ }^{1}$, Ismael Nyanquini ${ }^{1}$ and Stanislas Ouaro ${ }^{2^{*}}$

* Correspondence: ouaro@yahoo.fr ¿2Laboratoire d'Analyse

Mathématique des Equations (LAME) UFR. Sciences Exactes et Appliquées, Université de

Ouagadougou 03 BP 7021 Ouaga 03, Ouagadougou, Burkina Faso

Full list of author information is available at the end of the article

\section{Abstract}

In this article, we prove the existence and uniqueness of solutions for a family of discrete boundary value problems for data $f$ which belongs to a discrete Hilbert space $W$.

2010 Mathematics Subject Classification: 47A75; 35B38; 35P30; 34L05; 34L30.

Keywords: Discrete boundary value problem, critical point, weak solution, electrorheological fluids

\section{Introduction}

In this article, we study the following nonlinear Neumann discrete boundary value problem:

$$
\left\{\begin{array}{l}
-\Delta(a(k-1, \Delta u(k-1)))+|u(k)|^{p(k)} u(k)=f(k), \quad k \in \mathbb{Z}[1, T] \\
\Delta u(0)=\Delta u(T)=0,
\end{array}\right.
$$

where $T \geq 2$ is a positive integer, and $\Delta u(k)=u(k+1)-u(k)$ is the forward difference operator. Throughout this article, we denote by $\mathbb{Z}[a, b]$ the discrete interval $\{a, a+1$, $\ldots, b\}$, where $a$ and $b$ are integers and $a<b$.

We also consider the function space

$$
W=\{v: \mathbb{Z}[0, T+1] \rightarrow \mathbb{R} ; \text { such that } \Delta v(0)=\Delta v(T)=0\},
$$

where $W$ is a $\mathrm{T}$-dimensional Hilbert space $[1,2]$ with the inner product

$$
(u, v)=\sum_{k=1}^{T+1} \Delta u(k-1) \Delta v(k-1)+\sum_{k=1}^{T} u(k) v(k), \quad \forall u, v \in W .
$$

The associated norm is defined by

$$
\|u\|=\left(\sum_{k=1}^{T+1}|\Delta u(k-1)|^{2}+\sum_{k=1}^{T}|u(k)|^{2}\right)^{\frac{1}{2}}
$$

For the data $f$ and $a$, we assume the following:

$$
f: \mathbb{Z}[1, T] \rightarrow \mathbb{R}
$$




$$
\begin{aligned}
& \left\{\begin{array}{l}
a(k, .): \mathbb{R} \rightarrow \mathbb{R} \forall k \in \mathbb{Z}[0, T] \text { and there exists a mapping } \\
A: \mathbb{Z}[0, T] \times \mathbb{R} \rightarrow \mathbb{R} \text { which satisfies }: a(k, \xi)=\frac{\partial}{\partial \xi} A(k, \xi) \\
\text { and } A(k, 0)=0, \forall k \in \mathbb{Z}[0, T],
\end{array}\right. \\
& (a(k, \xi)-a(k, \eta)) .(\xi-\eta)>0 \forall k \in \mathbb{Z}[0, T] \text { and } \xi, \eta \in \mathbb{R} \text { such that } \xi \neq \eta,
\end{aligned}
$$

and

$$
|\xi|^{p(k)} \leq a(k, \xi) \xi \leq p(k) A(k, \xi) \forall k \in \mathbb{Z}[0, T] \text { and } \xi \in \mathbb{R} .
$$

Moreover, in this article, we assume that the function

$$
p: \mathbb{Z}[0, T] \rightarrow(2,+\infty) .
$$

The theory of difference equations occupy a central position in applicable analysis. We refer to the recent results of Agarwal et al. [1], Candito and D'Agui [2], Yu and Guo [3], Koné and Ouaro [4], Cai and Yu [5], Zhang and Liu [6], Mihailescu et al. [7], Cabada et al. [8] and the references therein. In [6], the authors studied the following problem:

$$
\left\{\begin{array}{l}
\Delta^{2} \gamma(k-1)+\lambda f(y(k))=0, \quad k \in \mathbb{Z}[1, T] \\
\gamma(0)=\gamma(T+1)=0
\end{array}\right.
$$

where $\lambda>0$ is a parameter, $\Delta^{2} y(k)=\Delta(\Delta y(k))$, and $f:[0,+\infty) \rightarrow \mathbb{R}$ a continuous function satisfying the condition

$$
f(0)=-a<0, \text { where } a \text { is a positive constant. }
$$

The problem (1.10) is referred as the "semipositone" problem in the literature, which was introduced by Castro and Shivaji [9]. Semipositone problems arise in bulking of mechanical systems, design of suspension bridges, chemical reactions, astrophysics, combustion, and management of natural resources.

The studies regarding problems like (1.1) or (1.10) can be placed at the interface of certain mathematical fields such as nonlinear partial differential equations and numerical analysis. On the other hand, they are strongly motivated by their applicability in mathematical physics as mentioned above.

In [2], Candito and D'Agui studied the following problem:

$$
\left\{\begin{array}{l}
-\Delta\left(\Phi_{p}\left(\Delta u_{k-1}\right)\right)+q_{k} \Phi_{p}\left(u_{k}\right)=\lambda f\left(k, u_{k}\right), \quad k \in[1, N] \\
\Delta u_{0}=\Delta u_{N}=0
\end{array}\right.
$$

where $N$ is a fixed positive integer, $[1, N]$ is the discrete interval $1, \ldots, N, q_{k}>0$ for all $k \in[1, N], \lambda$ is a positive real parameter, $\Phi_{p}(s):=|s|^{p-2} s, 1<p<+\infty$ and $f:[1, N] \times \mathbb{R}$ $\rightarrow \mathbb{R}$ is a continuous function. Candito and D'Agui proved the existence of three solutions for (1.12) by using a three critical points theorem by Jiang and Zhou [[10], Theorem 2.6].

In this article, we consider the same boundary conditions as in [2], but the main operator is more general than the one in [2] and involve variable exponent. 
Problem (1.1) is a discrete variant of the variable exponent anisotropic problem:

$$
\left\{\begin{array}{l}
-\sum_{i=1}^{N} \frac{\partial}{\partial x_{i}} a_{i}\left(x, \frac{\partial u}{\partial x_{i}}\right)=f(x) \text { in } \Omega \\
u=0 \text { on } \partial \Omega
\end{array}\right.
$$

where $\Omega \subset \mathbb{R}^{N}(N \geq 3)$ is a bounded domain with smooth boundary, $f \in L^{\infty}(\Omega), p_{i}$ continuous on $\bar{\Omega}$ such that $1<p_{i}(x)<N$ and $\sum_{i=1}^{N} \frac{1}{p_{i}^{-}}>1$ for all $x \in \bar{\Omega}$, and all $i \in \mathbb{Z}[1$, $N]$, where $p_{i}^{-}:=$ess $\inf _{x \in \Omega} p_{i}(x)$.

Problem (1.13) was recently analyzed by Koné et al. [11], Ouaro [12], and generalyzed to a Radon measure data by Koné et al. [13]. Problems like (1.13) have been intensively studied in the last few decades since they can model various phenomena arising from the study of elastic mechanics [14,15], electrorheological fluids [14,16-18], and image restoration [19]. In [19], Chen et al. studied a functional with variable exponent $1 \leq p(x) \leq 2$ which provides a model for image denoizing, enhancement and restoration. Their article created motivation for the study of problems with variable exponent.

Note that Mihailescu et al. [20,21] were the first authors to study anisotropic elliptic problems with variable exponent.

Our aim in this article is to use a minimization method to establish some existence results of solutions of (1.1). The idea of the proof is to transfer the problem of the existence of solutions for (1.1) into the problem of the existence of a minimizer for some associated energy functional. Let us point out that, to the best of our knowledge, discrete problems like (1.1) involving anisotropic exponents have been discussed for the first time by Mihailescu, Radulescu, and Tersian [7] and for the second time by Koné and Ouaro [4]. In [7], the authors proved by using critical point theory, the existence of a continuous spectrum of eigenvalues for the problem:

$$
\left\{\begin{array}{l}
-\Delta\left(|\Delta u(k-1)|^{p(k-1)-2} \Delta u(k-1)\right)=\lambda|u(k)|^{q(k)-2} u(k), \quad k \in \mathbb{Z}[1, T] \\
u(0)=u(T+1)=0,
\end{array}\right.
$$

where $T \geq 2$ is a positive integer, and the functions $p: \mathbb{Z}[0, T] \rightarrow[2, \infty)$ and $q: \mathbb{Z}[1$, $T] \rightarrow[2, \infty)$ are bounded while $\lambda$ is a positive constant.

In [4], Koné and Ouaro proved using minimization method the existence and uniqueness of weak solutions for the following problem:

$$
\left\{\begin{array}{l}
-\Delta(a(k-1, \Delta u(k-1)))=f(k), \quad k \in \mathbb{Z}[1, T] \\
u(0)=u(T+1)=0,
\end{array}\right.
$$

where $T \geq 2$ is a positive integer.

The function $a(k-1, \Delta u(k-1))$ which appears in the left-hand side of problem (1.1) is more general than the one which appears in (1.14). Indeed, as examples of functions which satisfy the assumptions (1.6)-(1.8), we can give the following:

- $A(k, \xi)=\frac{1}{p(k)}|\xi|^{p(k)}$, where $a(k, \xi)=|\xi|^{p(k)-2} \xi, \forall k \in \mathbb{Z}[0, T]$ and $\xi \in \mathbb{R}$.

- $A(k, \xi)=\frac{1}{p(k)}\left[\left(1+|\xi|^{2}\right)^{p(k) / 2}-1\right]$, where $a(k, \xi)=\left(1+|\xi|^{2}\right)^{(p(k)-2) / 2} \xi, \forall k \in \mathbb{Z}[0$,

$T]$ and $\xi \in \mathbb{R}$. 
In this article, the boundary condition is the Neumann one which is different to that in [4] which is the Dirichlet boundary condition.

The remaining part of this article is organized as follows. Sect. 2 is devoted to mathematical preliminaries. The main existence and uniqueness result is stated and proved in Sect. 3. Finally, in Sect. 4, we discuss some extensions.

\section{Preliminaries}

From now onwards, we will use the following notations:

$$
p^{-}=\min _{k \in \mathbb{Z}[0, T]} p(k) \text { and } p^{+}=\max _{k \in \mathbb{Z}[0, T]} p(k) .
$$

Moreover, it is useful to introduce other norms on $W$, namely

$$
|u|_{m}=\left(\sum_{k=1}^{T}|u(k)|^{m}\right)^{\frac{1}{m}} \forall u \in W \text { and } m \geq 2 .
$$

We have the following inequalities [5,7]:

$$
T^{(2-m) /(2 m)}|u|_{2} \leq|u|_{m} \leq T^{1 / m}|u|_{2} \forall u \in W \text { and } m \geq 2 .
$$

In the sequel, we will use the following auxiliary result:

\section{Lemma 2.1 .}

(a) There exist four positive constants $C_{1}, C_{2}, C_{3}$ and $C_{4}$ such that

$$
\begin{gathered}
\sum_{k=1}^{T+1}|\Delta u(k-1)|^{p(k-1)} \geq C_{1}\left(\sum_{k=1}^{T+1}|\Delta u(k-1)|^{2}\right)^{\frac{p^{-}}{2}}-C_{2}, \\
\sum_{k=1}^{T}|u(k)|^{p(k)} \geq C_{3}\left(\sum_{k=1}^{T}|u(k)|^{2}\right)^{\frac{p^{-}}{2}}-C_{4},
\end{gathered}
$$

$\forall u \in W$ with $\|u\|>1$.

(b) There exist two positive constants $C_{5}$ and $C_{6}$ such that

$$
\begin{gathered}
\sum_{k=1}^{T+1}|\Delta u(k-1)|^{p(k-1)} \geq C_{5}\left(\sum_{k=1}^{T+1}|\Delta u(k-1)|^{2}\right)^{\frac{p^{+}}{2}}, \\
\sum_{k=1}^{T}|u(k)|^{p(k)} \geq C_{6}\left(\sum_{k=1}^{T}|u(k)|^{2}\right)^{\frac{p^{+}}{2}},
\end{gathered}
$$

$\forall u \in W$ with $\|u\|<1$.

Proof Fix $u \in W$ with $\|u\|>1$, we define

$$
\alpha_{k}=\left\{\begin{array}{l}
p^{+} \text {if }|\Delta u(k)|<1 \\
p^{-} \text {if }|\Delta u(k)|>1
\end{array}\right.
$$

and

$$
\beta_{k}=\left\{\begin{array}{l}
p^{+} \text {if }|u(k)|<1 \\
p^{-} \text {if }|u(k)|>1,
\end{array}\right.
$$

for each $k \in \mathbb{Z}[0, T]$. 
(a) We deduce that

$$
\begin{aligned}
\sum_{k=1}^{T+1}|\Delta u(k-1)|^{p(k-1)} \geq & \sum_{k=1}^{T+1}|\Delta u(k-1)|^{\alpha_{k-1}} \\
\geq & \sum_{k=1}^{T+1}|\Delta u(k-1)|^{p^{-}} \\
& -\sum_{\left\{k \in \mathbb{Z}[1, T+1] ; \alpha_{k-1}=p_{+}\right\}}\left(|\Delta u(k-1)|^{p^{-}}-|\Delta u(k-1)|^{p^{+}}\right) \\
\geq & \sum_{k=1}^{T+1}|\Delta u(k-1)|^{p^{-}}-C_{2},
\end{aligned}
$$

for all $u \in W$ with $\|u\|>1$.

The inequality above and the relation (2.1) imply that

$$
\sum_{k=1}^{T+1}|\Delta u(k-1)|^{p(k-1)} \geq C_{1}\left(\sum_{k=1}^{T+1}|\Delta u(k-1)|^{2}\right)^{\frac{p}{2}}-C_{2},
$$

for all $u \in W$ with $\|u\|>1$.

Analogously, by using $\beta_{k}$ instead of $\alpha_{k}$, we prove that there exists $C_{3}$ and $C_{4}$ such that

$$
\sum_{k=1}^{T}|u(k)|^{p(k)} \geq C_{3}\left(\sum_{k=1}^{T}|u(k)|^{2}\right)^{\frac{p^{-}}{2}}-C_{4}
$$

for all $u \in W$ with $\|u\|>1$.

(b) We deduce as $|\Delta u(k)|<1$ and $|u(k)|<1$ since ||$u \| \mid<1$, that

$$
\sum_{k=1}^{T+1}|\Delta u(k-1)|^{p(k-1)} \geq \sum_{k=1}^{T+1}|\Delta u(k-1)|^{p^{+}} .
$$

and

$$
\sum_{k=1}^{T}|u(k)|^{p(k)} \geq \sum_{k=1}^{T}|u(k)|^{p^{+}} .
$$

We then get according to the two inequalities above and relation (2.1) that there exist two positive constants $C_{5}$ and $C_{6}$ such that

$$
\sum_{k=1}^{T+1}|\Delta u(k-1)|^{p(k-1)} \geq C_{5}\left(\sum_{k=1}^{T+1}|\Delta u(k-1)|^{2}\right)^{\frac{p^{+}}{2}}
$$

and

$$
\sum_{k=1}^{T}|u(k)|^{p(k)} \geq C_{6}\left(\sum_{k=1}^{T}|u(k)|^{2}\right)^{\frac{p^{+}}{2}}
$$

for all $u \in W$ such that $\|u\|<1 \square$ 


\section{Existence and uniqueness of weak solution}

In this section, we study the existence and uniqueness of weak solution of (1.1).

Definition 3.1 $A$ weak solution of (1.1) is a function $u \in W$ such that

$$
\sum_{k=1}^{T+1} a(k-1, \Delta u(k-1)) \Delta v(k-1)+\sum_{k=1}^{T}|u(k)|^{p(k)-2} u(k) v(k)=\sum_{k=1}^{T} f(k) v(k), \text { for any } v \in W .
$$

Note that since $W$ is a finite dimensional space, the weak solutions coincide with the classical solutions of problem (1.1).

We have the following result.

Theorem 3.2 Assume that (1.5)-(1.9) hold. Then, there exists a unique weak solution of (1.1).

The energy functional corresponding to problem (1.1), $J: W \rightarrow \mathbb{R}$ is defined by

$$
J(u)=\sum_{k=1}^{T+1} A(k-1, \Delta u(k-1))+\sum_{k=1}^{T} \frac{1}{p(k)}|u(k)|^{p(k)}-\sum_{k=1}^{T} f(k) u(k) .
$$

We first establish some basic properties of $J$.

Proposition 3.3 The functional $J$ is well defined on $W$ and is of class $C^{1}(W, \mathbb{R})$ with the derivative given by

$$
\left\langle J^{\prime}(u), v\right\rangle=\sum_{k=1}^{T+1} a(k-1, \Delta u(k-1)) \Delta v(k-1)+\sum_{k=1}^{T}|u(k)|^{p(k)-2} u(k) v(k)-\sum_{k=1}^{T} f(k) v(k),
$$

for all $u, v \in W$.

We define for $i=1, \ldots, N$ the functionals $I, \Lambda_{1}, \Lambda_{2} ; W \rightarrow \mathbb{R}$ by

$$
\begin{gathered}
I(u)=\sum_{k=1}^{T+1} A(k-1, \Delta u(k-1)), \\
\Lambda_{1}(u)=\sum_{k=1}^{T} \frac{1}{p(k)}|u(k)|^{p(k)}
\end{gathered}
$$

and

$$
\Lambda_{2}(u)=\sum_{k=1}^{T} f(k) u(k)
$$

The proof of Proposition 3.3 is contained in the following.

\section{Lemma 3.4}

(i) The functionals $I, \Lambda_{1}$ and $\Lambda_{2}$ are well defined on $W$.

(ii) The functionals $I, \Lambda_{1}$, and $\Lambda_{2}$ are of class $C^{1}(W, \mathbb{R})$ and

$$
\begin{aligned}
& \left\langle I^{\prime}(u), v\right\rangle=\sum_{k=1}^{T+1} a(k-1, \Delta u(k-1)) \Delta v(k-1), \\
& \left\langle\Lambda_{1}^{\prime}(u), v\right\rangle=\sum_{k=1}^{T}|u(k)|^{p(k)-2} u(k) v(k),
\end{aligned}
$$




$$
\left\langle\Lambda_{2}^{\prime}(u), v\right\rangle=\sum_{k=1}^{T} f(k) v(k)
$$

for all $u, v \in W$.

Proof

(i) $|I(u)|=\left|\sum_{k=1}^{T+1} A(k-1, \Delta u(k-1))\right|<+\infty$ since $A(k,$.$) is continuous for all k \in \mathbb{Z}$ $[0, T]$.

For all $k \in \mathbb{Z}[1, T]$,

$$
\left|\Lambda_{1}(u)\right|=\left.\left|\sum_{k=1}^{T} \frac{1}{p(k)}\right| u(k)\right|^{p(k)} \mid \leq \frac{1}{p_{-}}\left(\sum_{k=1}^{T}\left(|u(k)|^{p_{-}}+|u(k)|^{p_{+}}\right)\right)<+\infty .
$$

We also have by using Schwartz inequality that

$$
\left|\Lambda_{2}(u)\right|=\left|\sum_{k=1}^{T} f(k) u(k)\right| \leq \sum_{k=1}^{T}|f(k)||u(k)| \leq\left(\sum_{k=1}^{T}|f(k)|^{2}\right)^{\frac{1}{2}}\left(\sum_{k=1}^{T}|u(k)|^{2}\right)^{\frac{1}{2}}<+\infty .
$$

Then, $I, \Lambda_{1}$, and $\Lambda_{2}$ are well defined on $W$.

(ii) Clearly, $I, \Lambda_{1}$ and $\Lambda_{2}$ are in $C^{1}(W ; \mathbb{R})$. In what follows, we prove (3.4) and (3.5): choose $u, v \in W$. We have

$$
\begin{aligned}
\lim _{\delta \rightarrow 0^{+}} \frac{I(u+\delta v)-I(u)}{\delta} & =\lim _{\delta \rightarrow 0^{+}} \sum_{k=1}^{T+1} \frac{A(k-1, \Delta u(k-1)+\delta \Delta v(k-1))-A(k-1, \Delta u(k-1))}{\delta} \\
& =\sum_{k=1}^{T+1} \lim _{\delta \rightarrow 0^{+}} \frac{A(k-1, \Delta u(k-1)+\delta \Delta v(k-1))-A(k-1, \Delta u(k-1))}{\delta} \\
& =\sum_{k=1}^{T+1} a(k-1, \Delta u(k-1)) \Delta v(k-1) .
\end{aligned}
$$

Note also that there exists $v \in] 0,1[$ such that

$$
\begin{aligned}
& \frac{1}{\delta}\left(\frac{1}{p(k)}\left(|u(k)+\delta \varphi|^{p(k)}-|u(k)|^{p(k)}\right)\right)=|u(k)+v \delta \varphi|^{p(k)-2}(u(k)+v \delta \varphi) \varphi . \text { Then } \\
& \lim _{\delta \rightarrow 0^{+}} \frac{\Lambda_{1}(u+\delta v)-\Lambda_{1}(u)}{\delta}=\lim _{\delta \rightarrow 0^{+}} \sum_{k=1}^{T} \frac{1}{p(k)} \frac{|u(k)+\delta v(k)|^{p(k)}-|u(k)|^{p(k)}}{\delta} \\
&=\sum_{k=1}^{T}|u(k)|^{p(k)-2} u(k) v(k) .
\end{aligned}
$$

and

$$
\begin{aligned}
\lim _{\delta \rightarrow 0^{+}} \frac{\Lambda_{2}(u+\delta v)-\Lambda_{2}(u)}{\delta} & =\lim _{\delta \rightarrow 0^{+}} \sum_{k=1}^{T} \frac{f(k)(u(k)+\delta v(k))-f(k) u(k)}{\delta} \\
& =\sum_{k=1}^{T} f(k) v(k) .
\end{aligned}
$$


Lemma 3.5 The functional I is weakly lower semi-continuous.

Proof $A$ is convex with respect to the second variable according to (1.6). Thus, it is enough to show that $I$ is lower semi-continuous. For this, we fix $u \in W$ and $\varepsilon>0$. Since $I$ is convex, we deduce that for any $v \in W$

$$
\begin{aligned}
I(v) & \geq I(u)+\left\langle I^{\prime}(u), v-u\right\rangle \\
& \geq I(u)+\sum_{k=1}^{T+1} a(k-1, \Delta u(k-1))(\Delta v(k-1)-\Delta u(k-1)) \\
& \geq I(u)-\sum_{k=1}^{T+1}|a(k-1, \Delta u(k-1))||\Delta v(k-1)-\Delta u(k-1)| \\
& \geq I(u)-\left(\sum_{k=1}^{T+1}|a(k-1, \Delta u(k-1))|^{2}\right)^{\frac{1}{2}}\left(\sum_{k=1}^{T+1}|\Delta v(k-1)-\Delta u(k-1)|^{2}\right)^{\frac{1}{2}} \\
& \geq I(u)-\left(1+\sum_{k=1}^{T+1}|a(k-1, \Delta u(k-1))|^{2}\right)^{\frac{1}{2}}\|v-u\| \\
& \geq I(u)-\varepsilon,
\end{aligned}
$$

for all $v \in W$ with $\|v-u\|<\delta=\varepsilon / K(T, u)$, where $K(T, u)=\left(1+\sum_{k=1}^{T+1}|a(k-1, \Delta u(k-1))|^{2}\right)^{\frac{1}{2}}$.

Hence we conclude that $I$ is weakly lower semi-continuous.

Proposition 3.6 The functional $J$ is bounded from below, coercive, and weakly lower semi-continuous.

Proof By (1.8), we deduce that

$$
\begin{aligned}
J(u) & =\sum_{k=1}^{T+1} A(k-1, \Delta u(k-1))+\sum_{k=1}^{T} \frac{1}{p(k)}|u(k)|^{p(k)}-\sum_{k=1}^{T} f(k) u(k) \\
& \geq \sum_{k=1}^{T+1} \frac{1}{p(k)}|\Delta u(k-1)|^{p(k-1)}+\sum_{k=1}^{T} \frac{1}{p(k)}|u(k)|^{p(k)}-\sum_{k=1}^{T} f(k) u(k) .
\end{aligned}
$$

To prove the coercivity of $J$, we may assume that $\|u\|>1$ and we get from the above inequality, Lemma 2.1 and the fact that $p^{-}>2$ (then $x \mapsto x^{\frac{p^{-}}{2}}$ is convex), the following:

$$
\begin{aligned}
J(u) & \geq \frac{1}{p^{+}}\left(\sum_{k=1}^{T+1}|\Delta u(k-1)|^{p(k-1)}+\sum_{k=1}^{T}|u(k)|^{p(k)}\right)-\sum_{k=1}^{T} f(k) u(k) \\
& \geq \frac{C}{p^{+}}\left[\left(\sum_{k=1}^{T+1}|\Delta u(k-1)|^{2}\right)^{\frac{p^{-}}{2}}+\left(\sum_{k=1}^{T}|u(k)|^{2}\right)^{\frac{p^{-}}{2}}-C^{\prime}\right]-\left(\sum_{k=1}^{T}|f(k)|^{2}\right)^{\frac{1}{2}}\left(\sum_{k=1}^{T}|u(k)|^{2}\right)^{\frac{1}{2}} \\
& \geq \frac{C}{p^{+}}\|u\|^{p^{-}}-K_{1}\|u\|-K,
\end{aligned}
$$

where $K$ and $K_{1}$ are positive constants. Hence, since $p^{-}>1, J$ is coercive.

On the other hand, if $\|u\|<1$, we get by (1.8), Lemma 2.1 and the fact that $p^{+}>2$ (then $x \mapsto x^{\frac{p^{+}}{2}}$ is convex) the following: 


$$
\begin{aligned}
J(u) & \geq \frac{C}{p^{+}}\left[\left(\sum_{k=1}^{T+1}|\Delta u(k-1)|^{2}\right)^{\frac{p^{+}}{2}}+\left(\sum_{k=1}^{T}|u(k)|^{2}\right)^{\frac{p^{+}}{2}}\right]-K_{1}\|u\| \\
& \geq \frac{C}{p^{+}}\|u\|^{p^{+}}-K_{1}\|u\| \\
& \geq-K_{1} \\
& >-\infty .
\end{aligned}
$$

Therefore, $J$ is bounded from below.

As $I$ is weakly lower semi-continuous, $J$ is weakly lower semi-continuous. $\square$ We now give the proof of Theorem 3.2.

Proof of Theorem 3.2 By Proposition 3.6, $J$ has a minimizer which is a weak solution of (1.1).

In order to complete the proof of Theorem 3.2, we will prove the uniqueness of the weak solution.

Let $u_{1}$ and $u_{2}$ be two weak solutions of problem (1.1), then we have

$$
\sum_{k=1}^{T+1} a\left(k-1, \Delta u_{1}(k-1)\right) \Delta\left(u_{1}-u_{2}\right)(k-1)+\sum_{k=1}^{T}\left|u_{1}(k)\right|^{p(k)} u_{1}(k)\left(u_{1}-u_{2}\right)(k)=\sum_{k=1}^{T} f(k)\left(u_{1}-u_{2}\right)(k)
$$

and

$$
\sum_{k=1}^{T+1} a\left(k-1, \Delta u_{2}(k-1)\right) \Delta\left(u_{1}-u_{2}\right)(k-1)+\sum_{k=1}^{T}\left|u_{2}(k)\right|^{p(k)} u_{2}(k)\left(u_{1}-u_{2}\right)(k)=\sum_{k=1}^{T} f(k)\left(u_{1}-u_{2}\right)(k) .
$$

Adding (3.7) and (3.8), we obtain

$$
\left\{\begin{array}{l}
\sum_{k=1}^{T+1}\left[a\left(k-1, \Delta u_{1}(k-1)\right)-a\left(k-1, \Delta u_{2}(k-1)\right)\right] \Delta\left(u_{1}-u_{2}\right)(k-1) \\
+\sum_{k=1}^{T}\left[\left|u_{1}(k)\right|^{p(k)} u_{1}(k)-\left|u_{2}(k)\right|^{p(k)} u_{2}(k)\right]\left(u_{1}-u_{2}\right)(k)=0 .
\end{array}\right.
$$

Using (1.7), we deduce from (3.9) that

$$
\Delta u_{1}(k-1)=\Delta u_{2}(k-1) \text { for all } k=1, \ldots, T+1
$$

and

$$
u_{1}(k)=u_{2}(k) \text { for all } k=1, \ldots, T .
$$

Therefore,

$$
\left\|u_{1}-u_{2}\right\|=\left(\sum_{k=1}^{T+1}\left|\Delta\left(u_{1}-u_{2}\right)(k-1)\right|^{2}+\sum_{k=1}^{T}\left|\left(u_{1}-u_{2}\right)(k)\right|^{2}\right)^{\frac{1}{2}}=0,
$$

which implies that $u_{1}=u_{2}$. $\square$

\section{Some extensions}

\subsection{Extension 1}

In this section, we show that the existence result obtained for (1.1) can be extended to more general discrete boundary value problem of the form: 


$$
\left\{\begin{array}{l}
-\Delta(a(k-1, \Delta u(k-1)))+|u(k)|^{p(k)-2} u(k)=f(k, u(k)), \quad k \in \mathbb{Z}[1, T] \\
\Delta u(0)=\Delta u(T)=0,
\end{array}\right.
$$

where $T \geq 2$ is a positive integer and $f: \mathbb{Z}[1, T] \times \mathbb{R} \rightarrow \mathbb{R}$ is a continuous function with respect to the second variable for all $(k, z) \in \mathbb{Z}[1, T] \times \mathbb{R}$.

For every $k \in \mathbb{Z}[1, T]$ and every $t \in \mathbb{R}$, we put $F(k, t)=\int_{0}^{t} f(k, \tau) d \tau$.

By a weak solution of problem (4.1), we understand a function $u \in W$ such that

$$
\sum_{k=1}^{T+1} a(k-1, \Delta u(k-1)) \Delta v(k-1)+\sum_{k=1}^{T}|u(k)|^{p(k)-2} u(k) v(k)=\sum_{k=1}^{T} f(k, u(k)) v(k) \text {, for any } v \in W .
$$

We assume that there exist two positive constants $C_{7}$ and $C_{8}$ such that

$$
|f(k, t)| \leq C_{7}+C_{8}|t|^{\beta-1}, \text { for all }(k, t) \in \mathbb{Z}[1, T] \times \mathbb{R} \text {, where } 1<\beta<p^{-} .
$$

We have the following result:

Theorem 4.1 Under assumptions (1.6)-(1.9) and (4.3), the problem (4.1) has at least one weak solution.

Proof Let $g(u)=\sum_{k=1}^{T} F(k, u(k))$, then $g^{\prime}: W \rightarrow W$ is completely continuous and thus, $g$ is weakly lower semi-continuous.

Therefore, for $u \in W$,

$$
J(u)=\sum_{k=1}^{T+1} A(k-1, \Delta u(k-1))+\sum_{k=1}^{T} \frac{1}{p(k)}|u(k)|^{p(k)}-\sum_{k=1}^{T} F(k, u(k))
$$

is such that $J \in C^{1}(W ; \mathbb{R})$ and is weakly lower semi-continuous.

On the other hand, for all $u, v \in W$, we have

$$
\begin{aligned}
\lim _{\delta \rightarrow 0^{+}} \frac{g(u+\delta v)-g(u)}{\delta} & =\lim _{\delta \rightarrow 0^{+}} \sum_{k=1}^{T} \frac{F(k, u(k)+\delta v(k))-F(k, u(k))}{\delta} \\
& =\sum_{k=1}^{T} \lim _{\delta \rightarrow 0^{+}} \frac{F(k, u(k)+\delta v(k))-F(k, u(k))}{\delta} \\
& =\sum_{k=1}^{T} f(k, u(k)) v(k) .
\end{aligned}
$$

Consequently,

$$
\left\langle J^{\prime}(u), v\right\rangle=\sum_{k=1}^{T+1} a(k-1, \Delta u(k-1)) \Delta v(k-1)+\sum_{k=1}^{T}|u(k)|^{p(k)-2} u(k) v(k)-\sum_{k=1}^{T} f(k, u(k)) v(k),
$$

for all $u, v \in W$.

This implies that the weak solutions of problem (4.1) coincide with the critical points of $J$. Next, we prove that $J$ is bounded below and coercive complete the proof. From (4.3), we deduce that $|F(k, t)| \leq C\left(1+|t|^{\beta}\right)$ and then for $u \in W$ such that $\|u\|>1$, 


$$
\begin{aligned}
J(u) & \geq \frac{C}{p^{+}}\|u\|^{p_{-}}-C_{1}-\sum_{k=1}^{T} F(k, u(k)) \\
& \geq \frac{C}{p^{+}}\|u\|^{p_{-}}-C_{1}-C^{\prime} \sum_{k=1}^{T}\left(1+|u(k)|^{\beta}\right) \\
& \geq \frac{C}{p^{+}}\|u\|^{p_{-}}-C_{1}-C^{\prime} T-C^{\prime}\left(\sum_{k=1}^{T}|u(k)|^{\beta}\right) \\
& \geq \frac{C}{p^{+}}\|u\|^{p_{-}}-C_{1}-C^{\prime} T-C^{\prime \prime}\left(\sum_{k=1}^{T}|u(k)|^{2}\right)^{\frac{\beta}{2}} . \\
& \geq \frac{C}{p^{+}}\|u\|^{p_{-}}-C_{1}-C^{\prime} T-K\|u\|^{\beta} .
\end{aligned}
$$

Furthermore, by the fact that $1<\beta<p^{-}$, it turns out that

$$
J(u) \geq \frac{C}{p^{+}}\|u\|^{p_{-}}-C_{1}-C^{\prime} T-K\|u\|^{\beta} \rightarrow+\infty \text { as }\|u\| \rightarrow+\infty,
$$

where $K$ is a positive constant. Therefore, $J$ is coercive.

On the other hand, for $u \in W$ such that $\|u\|<1$,

$$
\begin{aligned}
J(u) & \geq \frac{C_{3}}{p^{+}}\|u\|^{p_{+}}-C T-K\|u\|^{\beta} \\
& \geq-C T-K\|u\|^{\beta} \\
& \geq-C T-K \\
& >-\infty .
\end{aligned}
$$

Hence, $J$ is bounded below. $\square$

Assume now that $F^{+}(k, t)=\int_{0}^{t} f^{+}(k, \tau) d \tau$ is such that there exist two positive constant $C_{9}$ and $C_{10}$ such that

$$
f^{+}(k, t) \leq C_{9}+C_{10}|t|^{\beta-1}, \text { for all }(k, t) \in \mathbb{Z}[1, T] \times \mathbb{R} \text {, where } 1<\beta<p^{-} .
$$

Then, we have the following result:

Theorem 4.2 Under assumptions (1.6)-(1.9) and (4.5), problem (4.1) has at least one weak solution.

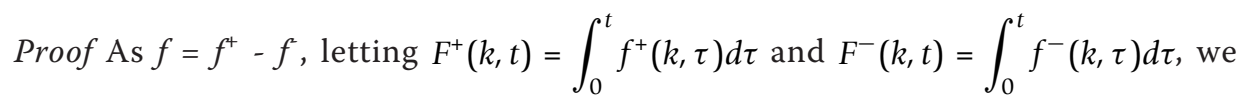
have

$$
\begin{aligned}
J(u) & =\sum_{k=1}^{T+1} A(k-1, \Delta u(k-1))+\sum_{k=1}^{T} \frac{1}{p(k)}|u(k)|^{p(k)}-\sum_{k=1}^{T} F(k, u(k)) \\
& =\sum_{k=1}^{T+1} A(k-1, \Delta u(k-1))+\sum_{k=1}^{T} \frac{1}{p(k)}|u(k)|^{p(k)}-\sum_{k=1}^{T} F^{+}(k, u(k))+\sum_{k=1}^{T} F^{-}(k, u(k)) \\
& \geq \sum_{k=1}^{T+1} A(k-1, \Delta u(k-1))+\sum_{k=1}^{T} \frac{1}{p(k)}|u(k)|^{p(k)}-\sum_{k=1}^{T} F^{+}(k, u(k)) .
\end{aligned}
$$

Therefore, similar to the proof of Theorem 4.1, Theorem 4.2 follows immediately. $\square$ 


\subsection{Extension 2}

In this section, we show that the existence result obtained for (1.1) can be extended to more general discrete boundary value problem of the form:

$$
\left\{\begin{array}{l}
-\Delta(a(k-1, \Delta u(k-1)))+|u(k)|^{p(k)-2} u(k)+\lambda|u(k)|^{\beta^{+}-2} u(k)=f(k, u(k)), \quad k \in \mathbb{Z}[1, T] \\
\Delta u(0)=\Delta u(T)=0,
\end{array}\right.
$$

where $T \geq 2$ is a positive integer, $\lambda \in \mathbb{R}^{+}$and $f: \mathbb{Z}[1, T] \times \mathbb{R} \rightarrow \mathbb{R}$ is a continuous function with respect to the second variable for all $(k, z) \in \mathbb{Z}[1, T] \times \mathbb{R}$.

For every $k \in \mathbb{Z}[1, T]$ and every $t \in \mathbb{R}$, we put $F(k, t)=\int_{0}^{t} f(k, \tau) d \tau$.

By a weak solution of problem (4.1), we understand a function $u \in W$ such that

$$
\left\{\begin{array}{l}
\sum_{k=1}^{T+1} a(k-1, \Delta u(k-1)) \Delta v(k-1)+\sum_{k=1}^{T}|u(k)|^{p(k)-2} u(k) v(k)+\lambda \sum_{k=1}^{T}|u(k)|^{\beta^{+}-2} u(k) v(k) \\
=\sum_{k=1}^{T} f(k, u(k)) v(k), \text { for any } v \in W .
\end{array}\right.
$$

We assume that there exist two positive constants $C_{11}$ and $C_{12}$ such that

$$
|f(k, t)| \leq C_{11}+C_{12}|t|^{\beta(k)-1}, \text { for all }(k, t) \in \mathbb{Z}[1, T] \times \mathbb{R} \text {, where } 1<\beta^{-}<p^{-} .
$$

We have the following result:

Theorem 4.3 Under assumptions (1.6)-(1.9) and (4.8), there exist $\lambda *>0$ such that for $\lambda \in\left[\lambda^{*},+\infty[\right.$, the problem (4.6) has at least one weak solution.

Proof Let $g(u)=\sum_{k=1}^{T} F(k, u(k))$, then $g^{\prime}: W \rightarrow W$ is completely continuous and thus, $g$ is weakly lower semi-continuous.

Therefore, for $u \in W$

$$
J(u)=\sum_{k=1}^{T+1} A(k-1, \Delta u(k-1))+\sum_{k=1}^{T} \frac{1}{p(k)}|u(k)|^{p(k)}+\frac{\lambda}{\beta^{+}} \sum_{k=1}^{T}|u(k)|^{\beta^{+}}-\sum_{k=1}^{T} F(k, u(k))
$$

is such that $J \in C^{1}(W ; \mathbb{R})$ and is weakly lower semi-continuous.

On the other hand, for all $u, v \in W$, we have

$$
\begin{aligned}
\lim _{\delta \rightarrow 0^{+}} \frac{g(u+\delta v)-g(u)}{\delta} & =\lim _{\delta \rightarrow 0^{+}} \sum_{k=1}^{T} \frac{F(k, u(k)+\delta v(k))-F(k, u(k))}{\delta} \\
& =\sum_{k=1}^{T} \lim _{\delta \rightarrow 0^{+}} \frac{F(k, u(k)+\delta v(k))-F(k, u(k))}{\delta} \\
& =\sum_{k=1}^{T} f(k, u(k)) v(k) .
\end{aligned}
$$

Consequently,

$$
\left\{\begin{array}{l}
\left\langle J^{\prime}(u), v\right\rangle=\sum_{k=1}^{T+1} a(k-1, \Delta u(k-1)) \Delta v(k-1)+\sum_{k=1}^{T}|u(k)|^{p(k)-2} u(k) v(k) \\
+\lambda \sum_{k=1}^{T}|u(k)|^{\beta^{+}-2} u(k) v(k)-\sum_{k=1}^{T} f(k, u(k)) v(k)
\end{array}\right.
$$

for all $u, v \in W$.

This implies that the weak solutions of problem (4.6) coincide with the critical points of $J$. We then have to prove that $J$ is bounded below and coercive complete the proof. 
From (4.8), we deduce that $\mid F(k, t) \leq C\left(1+|t|^{\beta(k)-1}\right)$ and then for $u \in W$ such that $\|u\|$ $>1$,

$$
\begin{aligned}
J(u) & \geq \frac{C}{p^{+}}\|u\|^{p_{-}}+\frac{\lambda}{\beta^{+}} \sum_{k=1}^{T}|u(k)|^{\beta^{+}}-C_{1}-\sum_{k=1}^{T} F(k, u(k)) \\
& \geq \frac{C}{p^{+}}\|u\|^{p_{-}}+\frac{\lambda}{\beta^{+}} \sum_{k=1}^{T}|u(k)|^{\beta^{+}}-C_{1}-C^{\prime} \sum_{k=1}^{T}\left(1+|u(k)|^{\beta(k)}\right) \\
& \geq \frac{C}{p^{+}}\|u\|^{p_{-}}+\frac{\lambda}{\beta^{+}} \sum_{k=1}^{T}|u(k)|^{\beta^{+}}-C_{1}-C^{\prime} T-C^{\prime}\left(\sum_{k=1}^{T}|u(k)|^{\beta(k)}\right) \\
& \geq \frac{C}{p^{+}}\|u\|^{p_{-}}+\frac{\lambda}{\beta^{+}} \sum_{k=1}^{T}|u(k)|^{\beta^{+}}-C_{1}-C^{\prime} T-C^{\prime}\left(\sum_{k=1}^{T}|u(k)|^{\beta^{-}}+\sum_{k=1}^{T}|u(k)|^{\beta^{+}}\right) \\
& \geq \frac{C}{p^{+}}\|u\|^{p_{-}}+\left(\frac{\lambda}{\beta^{+}}-C^{\prime}\right) \sum_{k=1}^{T}|u(k)|^{\beta^{+}}-C_{1}-C^{\prime} T-K\|u\|^{\beta^{-}} \\
& \geq \frac{C}{p^{+}}\|u\|^{p_{-}}-C_{1}-C^{\prime} T-K\|u\|^{\beta^{-}},
\end{aligned}
$$

where we put $\lambda^{*}=C \beta^{+}$.

Furthermore, by the fact that $1<\beta^{-}<p^{-}$, it turns out that

$$
J(u) \geq \frac{C}{p^{+}}\|u\|^{p_{-}}-C_{1}-C^{\prime} T-K\|u\|^{\beta^{-}} \rightarrow+\infty \text { as }\|u\| \rightarrow+\infty,
$$

where $K$ is a positive constant. Therefore, $J$ is coercive.

On the other hand, for $u \in W$ such that $\|u\|<1$,

$$
\begin{aligned}
J(u) & \geq \frac{C_{3}}{p^{+}}\|u\|^{p_{+}}-C^{\prime} T-K\|u\|^{\beta} \\
& \geq-C^{\prime} T-K\|u\|^{\beta} \\
& \geq-C^{\prime} T-K \\
& >-\infty .
\end{aligned}
$$

Hence, $J$ is bounded below.

\section{Acknowledgements}

The authors express their deepest thanks to the editor and anonymous referee for their comments and suggestions on the article.

\section{Author details}

${ }^{1}$ Laboratoire d'Analyse Mathématique des Equations (LAME) Institut des Sciences Exactes et Appliquées Université Polytechnique de Bobo Dioulasso 01 BP 1091 Bobo-Dioulasso 01, Bobo Dioulasso, Burkina Faso ²Laboratoire d'Analyse Mathématique des Equations (LAME) UFR. Sciences Exactes et Appliquées, Université de Ouagadougou 03 BP 7021 Ouaga 03, Ouagadougou, Burkina Faso

\section{Authors' contributions}

AG carried out Extension 1 and performed the introduction. IN carried out Extension 2 and performed the introduction. SO conceived the study, carried out the existence and uniqueness of weak solution, references and performed the final version of the paper.

All authors read and approved the final manuscript.

\section{Competing interests}

The authors declare that they have no competing interests. 


\section{References}

1. Agarwal, RP, Perera, K, O'Regan, D: Multiple positive solutions of singular and non-singular discrete problems via variational methods. Nonlinear Anal. 58,69-73 (2004). doi:10.1016/j.na.2003.11.012

2. Candito, P, D'Agui, G: Three solutions for a discrete nonlinear Neumann problem involving p-Laplacian. Adv Differ Equ 11 (2010). Article ID 862016

3. Yu, J, Guo, Z: On boundary value problems for a discrete generalized Emden-Fowler equation. J Math Anal Appl. 231, 18-31 (2006)

4. Koné, B, Ouaro, S: Weak solutions for anisotropic discrete boundary value problems. J Differ Equ Appl. 16(2), 1-11 (2010)

5. Cai, X, Yu, J: Existence theorems for second-order discrete boundary value problems. J Math Anal Appl. 320, 649-661 (2006). doi:10.1016/j.jmaa.2005.07.029

6. Zhang, G, Liu, S: On a class of semipositone discrete boundary value problem. J Math Anal Appl. 325, 175-182 (2007) doi:10.1016/j.jmaa.2005.12.047

7. Mihailescu, M, Radulescu, V, Tersian, S: Eigenvalue problems for anisotropic discrete boundary value problems. J Differ Equ Appl. 15(6), 557-567 (2009). doi:10.1080/10236190802214977

8. Cabada, A, lannizzoto, A, Tersian, S: Multiple solutions for discrete boundary value problems. J Math Anal Appl. 356 418-428 (2009). doi:10.1016/j.jmaa.2009.02.038

9. Castro, A, Shivaji, R: Nonnegative solutions for a class of radically symmetric non-positone problems. Proc Am Math Soc $106,735-740$ (1989)

10. Jiang, L, Zhou, Z: Three solutions to Dirichlet boundary value problems for $p$-Laplacian Difference equations. Adv Differ Equ 10 (2008). Article ID 345916

11. Koné, B, Ouaro, S, Traoré, S: Weak solutions for anisotropic nonlinear elliptic equations with variable exponent. Electron J Differ Equ. 2009(144), 1-11 (2009)

12. Ouaro, S: Well-posedness results for anisotropic nonlinear elliptic equations with variable exponent and $L^{1}$-data. Cubo J. 12(1), 133-148 (2010). doi:10.4067/S0719-06462010000100012

13. Koné, B, Ouaro, S, Soma, S: Weak solutions for anisotropic nonlinear elliptic problem with variable exponent and measure data. Int J Evol Equ. 5(3), 1-24 (2011)

14. Ruzicka, M: Electrorheological Fluids: Modelling and Mathematical Theory. Lecture Notes in Mathematics 1748. Springer Berlin (2002)

15. Zhikov, V: Averaging of functionals in the calculus of variations and elasticity. Math USSR Izv. 29, 33-66 (1987). doi:10.1070/IM1987v029n01ABEH000958

16. Diening, L: Theoretical and numerical results for electrorheological fluids. Ph.D. thesis. University of Freiburg, Germany (2002)

17. Halsey, TC: Electrorheological fluids. Science. 258, 761-766 (1992). doi:10.1126/science.258.5083.761

18. Rajagopal, KR, Ruzicka, M: Mathematical modeling of electrorheological materials. Contin Mech Thermodyn. 13, 59-78 (2011)

19. Chen, Y, Levine, S, Rao, M: Variable exponent, linear growth functionals in image processing. SIAM J Appl Math. 66(4), 1383-1406 (2006). doi:10.1137/050624522

20. Mihailescu, M, Pucci, P, Radulescu, V: Nonhomogeneous boundary value problems in anisotropic Sobolev spaces. C R Acad Sci Paris Ser I. 345, 561-566 (2007). doi:10.1016/j.crma.2007.10.012

21. Mihailescu, M, Pucci, P, Radulescu, V: Eigenvalue problems for anisotropic quasi-linear elliptic equations with variable exponent. J Math Anal Appl. 340,687-698 (2008). doi:10.1016/j.jmaa.2007.09.015

doi:10.1186/1687-1847-2011-32

Cite this article as: Guiro et al:: On the solvability of discrete nonlinear Neumann problems involving the $p(x)$ Laplacian. Advances in Difference Equations 2011 2011:32.

\section{Submit your manuscript to a SpringerOpen ${ }^{\circ}$ journal and benefit from:}

- Convenient online submission

Rigorous peer review

- Immediate publication on acceptance

- Open access: articles freely available online

- High visibility within the field

- Retaining the copyright to your article

Submit your next manuscript at $\boldsymbol{s p r i n g e r o p e n . c o m ~}$ 